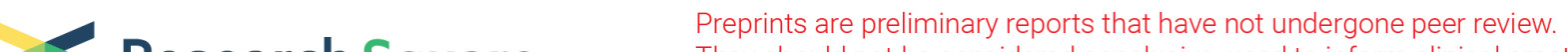 Research Square
}

\section{Ultrasound-Based Radiomics Analysis for Preoperative Prediction of Central and Lateral Cervical Lymph Node Metastasis in Papillary Thyroid Carcinoma: A Multi-Institutional Study}

\section{Yuyang Tong}

Fudan University Shanghai Cancer Center, Fudan University

Jingwen Zhang

Ruijin Hospital, Shanghai Jiao Tong University Medical School

Yi Wei

Fudan University Shanghai Cancer Center, Fudan University

Jinhua Yu

Fudan University

Weiwei Zhan

Ruijin Hospital, Shanghai Jiao Tong University Medical School

Hansheng Xia

Zhongshan Hospital, Fudan University, China

Shichong Zhou ( $\nabla$ sczhou@hotmail.com )

Fudan University Shanghai Cancer Center, Fudan University

\section{Yuanyuan Wang}

Fudan University

\section{Cai Chang}

Fudan University Shanghai Cancer Center, Fudan University

\section{Research Article}

Keywords: Papillary thyroid carcinoma, Ultrasound, Radiomics, Nomogram, Cervical lymph node metastasis

Posted Date: February 1st, 2022

DOI: https://doi.org/10.21203/rs.3.rs-1257799/v1

License: (9) This work is licensed under a Creative Commons Attribution 4.0 International License. Read Full License 


\section{Abstract \\ Background}

An accurate preoperative assessment of cervical lymph node metastasis (LNM) is important for choosing optimal therapeutic strategy for papillary thyroid carcinoma (PTC) patients. This study aimed to develop and validate two ultrasound (US) nomograms for the individual prediction of central and lateral compartment LNM in patients with PTC.

\section{Methods}

A total of 720 PTC patients from 3 institutions were enrolled in this study. They were categorized into a primary cohort, an internal validation, and two external validation cohorts. Radiomics features were extracted from conventional US images. LASSO regression was used to select optimized features to construct the radiomics signature. Two nomograms integrating independent clinical variables and radiomics signature were established with multivariate logistic regression. The performance of the nomograms was assessed with regard to discrimination, calibration, and clinical usefulness.

\section{Results}

The radiomics scores were significantly higher in patients with central/lateral LNM. A radiomics nomogram indicated good discrimination for central compartment LNM, with an area under the curve (AUC) of 0.875 in the training set, the corresponding value in the validation sets were $0.856,0.870$ and 0.870 , respectively. Another nomogram for predicting lateral LNM also demonstrated good performance with a training AUC of 0.938 , an internal validation AUC of 0.905 , and two external validation AUC of 0.881 and 0.903 , respectively. The clinical utility of the nomograms was confirmed by the decision curve analysis.

\section{Conclusion}

The nomograms proposed here have favorable performance for preoperatively predicting cervical LNM, and hold promise for optimizing the personalized treatment, which may improve the survival outcome in PTC patients.

\section{Introduction}

Papillary thyroid carcinoma (PTC) accounts for approximately $85 \%$ of all thyroid carcinoma cases, and its global incidence is increasing over the past few decades(1). Despite having an indolent clinical course, PTC is associated with cervical lymph node metastasis (LNM)(2). Cervical LNM occurs in approximately $30-80 \%$ of PTC cases and is one of the most important risk factors for locoregional recurrence(3). The 
presence of LNM also affects the staging and treatment options for PTC(4). Central compartment LNM is usually treated with central compartment neck dissection (CCND), and central LNM has been found in $45 \%$ of PTC patients with clinically negative LNs (cN0) who undergo prophylactic CCND(5). However, the risk of hypoparathyroidism and recurrent laryngeal nerve injury is heightened with CCND, whether CCND should be performed prophylactically in cNO patients remains controversial(6). Patients with preoperatively evident lateral nodal metastasis typically require aggressive treatment, including lateral compartment lymph node (LN) dissection and high-dose radioactive iodine (RAI) therapy(7). However, therapeutic neck dissection and/or RAI ablation increases the morbidity and reduces the quality of life(8). The reduction of the incidence of complication must be based on reduction of unnecessary CLN dissection. Therefore, an accurate preoperative assessment of cervical LNM is important for choosing the optimal therapeutic strategy for PTC patients.

Ultrasound (US) is the preferred screening modality for preoperative assessment of cervical LN (CLN) status in PTC patients(9). However, preoperative US has a low sensitivity (38-59\%) for detecting cervical $\operatorname{LNM}(6,10)$. Furthermore, the diagnostic performance of neck US differs among physicians and the interobserver variability is high(11). Fine-needle aspiration (FNA) is an invasive approach and its sensitivity in evaluating LNM varies and is specific to operator(12). Thus, a non-invasive, effective alternative imaging modality with improved accuracy is urgently needed in clinical practice.

Fueled by the rapid advances in medical imaging and developments in analytical methods, the field of radiomics has attracted increasing research attention in recent years(13). Radiomics refers to the highthroughput extraction of extensive quantitative features to convert medical images into mineable data that could likely be used as diagnostic, predictive or prognostic biomarkers and support the clinical decision-making system(14). Accordingly, our previous studies have proved the value of radiomics in predicting the cervical LNM of PTC(15-17). To our knowledge, the US radiomics method applied in individually predicting cervical central and lateral LNM for PTC patients, respectively, has not been reported yet.

Therefore, the current study aimed to develop and validate nomograms which incorporated the US radiomics as well as the clinical risk factors for individual prediction of central and lateral cervical LNM in PTC patients.

\section{Materials And Methods}

\section{Patients}

Between January 2019 to June 2019, consecutive patients with thyroid nodules in hospital 1 (Fudan University Shanghai Cancer Center, Shanghai, China; training and validation cohort 1), hospital 2 (Zhongshan Hospital, Fudan University, Shanghai, China; validation cohort 2) and hospital 3 (Ruijin Hospital, Shanghai Jiao Tong University, Shanghai, China; validation cohort 3) were included. The inclusion criteria were as follows: ( $\mathbb{(})$ primary surgical resection was performed for the target tumor which had a pathological diagnosis of PTC; ( $(\mathbb{Z})$ preoperative neck US was performed, and the images were 
recorded and saved as DICOM format; ( $)$ US images meet the requirements for the stored images(15); ( $)$ patients with complete clinicopathological information. The exclusion criteria included the following: (区)

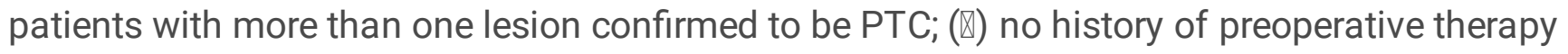
(radiofrequency or microwave ablation, neck radiation therapy); (囚) patients with only microscopic cervical LNM. After that, a total of 720 patients were enrolled in this study. Four hundred forty-three patients were chronologically divided into two cohorts: the training cohort with 300 patients who were treated between January and April 2019, and the internal validation cohort with 143 patients who were treated between May and June 2019. Two external validation cohorts of 144 and 133 consecutive patients, respectively, were included using the same criteria. The patients were divided into two groups based on the pathological reports of the lymph node status after CLN dissection.

This retrospective study was approved by the Ethics Committees of all participating hospitals (Shanghai Cancer Center, Ruijin Hospital, and Zhongshan Hospital), and performed in accordance with the Declaration of Helsinki. The inform consent was waived because of the retrospective nature of the study.

\section{Surgery and Pathology}

All patients received either total thyroidectomy or lobectomy in accordance with the clinical TNM staging, and underwent therapeutic or prophylactic central compartment dissection. Lateral neck LN dissection was performed for patients with FNA proven, or radiologically suspicious LNs that were not suitable for FNA. The resected thyroid and LNs specimen were collected and subjected to pathological examination, including the determination of unifocal or multifocal, and tumor size (maximum diameter of the tumor); the number, size and neck level of the metastatic LNs. The pathological results of the LNs were used as the gold standard(15).

\section{Clinical characteristics}

The baseline clinical information, including age, gender, thyroglobulin (TG), thyroid stimulating hormone (TSH), thyroglobulin antibody (TGAB), thyroid peroxidase antibody (TPOAB), and cytological findings of FNA were collected from the medical record system. The results of TG, TSH, TGAB, and TPOAB were detected within one week prior to surgical treatment. According to the previous reports and clinical experience $(18,19)$, the threshold set for TG, TSH, TGAB, and TPOAB were as follows: $T G \geq 77 \mathrm{ng} / \mathrm{ml}$, $T S H \geq 2.5 \mathrm{ng} / \mathrm{ml}, T G A B \geq 100 \mathrm{IU} / \mathrm{ml}$, and $T P O A B \geq 35 \mathrm{IU} / \mathrm{ml}$. Imaging data including US images, US reports and CT reports were obtained from medical image station.

\section{US image acquisition and US-reported CLNs status}

All patients received neck US examination before surgery. US images were acquired with a Supersonic Aixplorer System using a 15-4 linear-array transducer (SuperSonic Imaging, Fracnce) by radiologists with more than 6 years of experience. The US acquisition parameters were consistent among patients: image depth, $3 \mathrm{~cm}$; focus parallel to the lesion, and gain $53 \%$, and the spatial resolution of axial and lateral was $0.2 \mathrm{~mm}$ and $0.4 \mathrm{~mm}$, respectively(20). The detailed requirements for the US images were described in our 
previous study(20). The diagnostic result "central LNM" or "lateral LNM" in the US report was defined as US-reported central/lateral cervical LNM positive. The results of "LNs negative", "inflammatory LNs" and "small LNs" were categorized as US-reported central/lateral cervical LNM negative $(15,20)$. The abnormal US finding suggestive of LNM include the following: ( () round shape; ( $($ )absence of the fatty hilum; $(\mathbb{\nabla})$ microcalcification within the nodes; ( $\nabla)$ presence of peripheral flow; (v) heterogeneity with cystic components(21). LNs that met two or more of the five criteria would be considered positive. The diagnostic sensitivity, specificity and accuracy of neck US examination in the training and validation sets were calculated based upon the US reports(15).

\section{Region of interest segmentation and feature extraction}

A representative US image were selected, in which the tumor was manually segmented by 2 senior US physicians with more than 6-year-experience in thyroid US imaging who were blinded from the final LNs status. The MATLAB software written by our team was used to extract radiomics features (MATLAB R2015b, Mathworks). To evaluate the reproducibility of feature extraction, fifty cases were randomly selected and double-blinded for comparing manual segmentation by two physicians. The interclass correlation coefficient (ICC) was calculated to measure the intra-observer and inter-observer agreement of the radiomics feature extraction. An ICC greater than 0.80 was considered to indicated good agreement.

\section{Feature selection, radiomics signature building, and validation}

The detailed procedure of radiomics feature selection was described in our previous study(15). The selected radiomics features were then combined to build a radiomics signature. Based on the estimated coefficient, a radiomics score (Rad-score) for each case was computed to reflect the risk of cervical central and lateral LNM. The association between US radiomics signature and central/lateral LNs status was assessed in the training cohort by Mann-Whitney $\mathrm{U}$ test before being subjected to the validation cohorts.

\section{Development of US radiomics nomogram}

Student's $t$ test (continuous variables) or $\mathrm{c}^{2}$ test (categorical variables) was employed to identified the clinicopathological factors associated with central and lateral cervical LNM. A multivariate logistic regression analysis comprising the Rad-score and the independent risk factors was conducted. The backward stepwise selection procedure was utilized with a liberal $p$ value less than 0.05 as the retention criteria to select the final independent predictors for central/lateral LNM. Then a radiomics nomogram was developed based on the multivariate analysis in the training set.

\section{Performance of the US radiomics nomogram}

The receiver operating characteristic curve (ROC) of the US-reported LN status, radiomics signature, and radiomics nomogram in each cohort was plotted. The discriminatory performance of the three models was evaluated using the area under curve (AUC). Calibration of the US radiomics nomogram in all four 
cohorts was assessed by the calibration curve and Hosmer-Lemeshow test. To determine the clinical usefulness of the US radiomics nomogram, decision curve analysis (DCA) was performed by quantifying the net benefits at different threshold probabilities in our entire dataset.

\section{Statistical analysis}

The statistical analyses were performed with R software (version 3.5.3 http://www.r-project.prg), where the package 'shiny', 'foreign', 'nomogramFormula', 'tmcn', 'rms', 'set', 'glmnet', 'rmda' and 'DT' were applied. All other statistical tests were conducted using SPSS software (version 26, Chicago, IL). The categorical and continuous variables were presented as frequency (percentage) and mean \pm standard deviation (SD), respectively. Categorical variables were compared by $c^{2}$ test; student's t-test was used for comparison between continuous variables. The Delong test was employed to compare different AUC. A two-sided $p<0.05$ was considered to indicate statistically significant.

\section{Results}

\section{Clinical characteristics}

The clinicopathological characteristics of the patients in the entire dataset were presented in Table 1 and 2. The positive rate in terms of the central LNM in the training and validation cohorts were $34.7 \%, 32.9 \%$, $48.6 \%$, and $31.6 \%$, respectively; and $9.0 \%, 10.5 \%, 10.4 \%$, and $14.3 \%$, respectively, with regard to the lateral LNM. No significant differences in the positive rate were found among the four cohorts $(p=0.058$; $p=0.435)$. 
Table 1

Associations between the central CLN status and clinical parameters in the training and validation cohorts

\begin{tabular}{|c|c|c|c|c|c|c|}
\hline \multirow[b]{2}{*}{ Characteristic } & \multicolumn{3}{|c|}{ Training cohort $(n=300)$} & \multicolumn{3}{|c|}{$\begin{array}{l}\text { Validation cohort } 1 \\
(n=143)\end{array}$} \\
\hline & $\begin{array}{l}\text { CLNM }+ \\
(n=104)\end{array}$ & $\begin{array}{l}\text { CLNM - } \\
(n=196)\end{array}$ & $p$ & $\begin{array}{l}\text { CLNM + } \\
(n=47)\end{array}$ & $\begin{array}{l}\text { CLNM - } \\
(n=96)\end{array}$ & $p$ \\
\hline Age (years) & & & $<0.001$ & & & 0.038 \\
\hline Mean \pm SD & $38.9 \pm 10.6$ & $45.3 \pm 11.4$ & & $\begin{array}{l}39.0 \pm \\
11.4\end{array}$ & $\begin{array}{l}43.6 \pm \\
12.6\end{array}$ & \\
\hline Age (years) & & & $<0.001$ & & & 0.011 \\
\hline$>45$ & $28(26.9)$ & $94(48.0)$ & & $13(27.7)$ & $48(50.0)$ & \\
\hline$\leq 45$ & $76(73.1)$ & $102(52.0)$ & & $34(72.3)$ & $48(50.0)$ & \\
\hline Gender (\%) & & & 0.623 & & & 0.157 \\
\hline Male & $32(30.8)$ & $55(28.1)$ & & $16(34.0)$ & $22(22.9)$ & \\
\hline Female & $72(69.2)$ & $141(71.9)$ & & $31(66.0)$ & $74(77.1)$ & \\
\hline TSH (\%) & & & 0.487 & & & 0.975 \\
\hline Normal & $93(89.4)$ & $180(91.8)$ & & 44 (93.6) & $90(93.8)$ & \\
\hline Abnormal & $11(10.6)$ & $16(8.2)$ & & $3(6.4)$ & $6(6.3)$ & \\
\hline TG (\%) & & & 0.143 & & & 0.482 \\
\hline Normal & $92(88.5)$ & $183(93.4)$ & & 45 (95.7) & $89(92.7)$ & \\
\hline Abnormal & $12(11.5)$ & $13(6.6)$ & & $2(4.3)$ & $7(7.2)$ & \\
\hline TGAB (\%) & & & 0.518 & & & 0.111 \\
\hline Normal & $64(61.5)$ & $128(65.3)$ & & $27(57.4)$ & $68(70.8)$ & \\
\hline Abnormal & $40(38.5)$ & $68(34.7)$ & & $20(42.6)$ & $28(29.2)$ & \\
\hline TPOAB (\%) & & & 0.091 & & & 0.102 \\
\hline Normal & $69(66.3)$ & $148(75.5)$ & & 42 (89.4) & $75(78.1)$ & \\
\hline Abnormal & 35 (33.7) & $48(24.5)$ & & $5(10.6)$ & $21(21.9)$ & \\
\hline Tumor size (mm) & & & $<0.001$ & & & $<0.001$ \\
\hline
\end{tabular}

CLN, cervical lymph node; CLNM, cervical lymph metastasis; TG, thyroglobulin; TGAB, thyroglobulin antibody; TPOAB, thyroid peroxidase antibody; TSH, thyroid stimulating hormone; LNM, lymph node metastasis; SD, standard deviation; US, ultrasound. Significant differences are highlighted in boldface. 


\begin{tabular}{|c|c|c|c|c|c|c|}
\hline \multirow[b]{2}{*}{ Mean \pm SD } & \multicolumn{3}{|c|}{ Training cohort $(n=300)$} & \multicolumn{3}{|c|}{$\begin{array}{l}\text { Validation cohort } 1 \\
(n=143)\end{array}$} \\
\hline & $13.4 \pm 8.1$ & $9.2 \pm 5.9$ & & $\begin{array}{l}13.9 \pm \\
10.1\end{array}$ & $9.0 \pm 7.6$ & \\
\hline Tumor size $(\mathrm{mm})$ & & & $<0.001$ & & & $<0.001$ \\
\hline$\geq 1$ & $64(61.5)$ & $53(27.0)$ & & $29(61.7)$ & $15(15.6)$ & \\
\hline$<1$ & $40(38.5)$ & $143(73.0)$ & & $18(38.3)$ & $81(84.4)$ & \\
\hline $\begin{array}{l}\text { US-reported central } \\
\text { CLN status (\%) }\end{array}$ & & & $<0.001$ & & & $<0.001$ \\
\hline negative & $52(50.0)$ & $177(90.3)$ & & $24(51.1)$ & $87(90.6)$ & \\
\hline positive & $52(50.0)$ & $19(9.7)$ & & $23(48.9)$ & $9(9.4)$ & \\
\hline $\begin{array}{l}\text { Radiomics score } \\
\text { Median }\end{array}$ & -0.2873 & -0.4266 & $<0.001$ & -0.2631 & -0.4063 & $<0.001$ \\
\hline \multirow[t]{2}{*}{ (interquartile range) } & $\begin{array}{l}(-0.4481 \\
-0.1983)\end{array}$ & $\begin{array}{l}(-0.5547 \\
-0.3014)\end{array}$ & & $\begin{array}{l}(-0.4026 \\
-0.1533)\end{array}$ & $\begin{array}{l}(-0.5130 \\
-0.2765)\end{array}$ & \\
\hline & \multicolumn{3}{|c|}{$\begin{array}{l}\text { Validation cohort } 2 \\
(n=144)\end{array}$} & \multicolumn{3}{|c|}{$\begin{array}{l}\text { Validation cohort } 3 \\
(n=133)\end{array}$} \\
\hline Characteristic & $\begin{array}{l}\text { CLNM + } \\
(n=70)\end{array}$ & $\begin{array}{l}\text { CLNM - } \\
(n=74)\end{array}$ & $p$ & $\begin{array}{l}\text { CLNM + } \\
(n=42)\end{array}$ & $\begin{array}{l}\text { CLNM - } \\
(n=91)\end{array}$ & $p$ \\
\hline Age (years) & & & 0.017 & & & 0.014 \\
\hline Mean \pm SD & $37.3 \pm 10.9$ & $41.9 \pm 11.9$ & & $\begin{array}{l}36.7 \pm \\
12.1\end{array}$ & $\begin{array}{l}45.2 \pm \\
11.8\end{array}$ & \\
\hline Age (years) & & & 0.052 & & & 0.018 \\
\hline$>45$ & $22(31.4)$ & $35(47.3)$ & & $12(28.6)$ & $46(50.5)$ & \\
\hline$\leq 45$ & $48(68.6)$ & $39(52.7)$ & & $30(71.4)$ & $45(49.5)$ & \\
\hline Gender (\%) & & & 0.336 & & & 0.897 \\
\hline Male & $20(28.6)$ & $16(21.6)$ & & $12(28.6)$ & $27(29.7)$ & \\
\hline Female & $50(71.4)$ & $58(78.4)$ & & $30(71.4)$ & $64(70.3)$ & \\
\hline TSH (\%) & & & 0.920 & & & 0.551 \\
\hline Normal & $64(91.4)$ & $68(91.9)$ & & $38(90.5)$ & $85(93.4)$ & \\
\hline
\end{tabular}

CLN, cervical lymph node; CLNM, cervical lymph metastasis; TG, thyroglobulin; TGAB, thyroglobulin antibody; TPOAB, thyroid peroxidase antibody; TSH, thyroid stimulating hormone; LNM, lymph node metastasis; SD, standard deviation; US, ultrasound. Significant differences are highlighted in boldface. 


\begin{tabular}{|c|c|c|c|c|c|c|}
\hline \multirow[b]{2}{*}{ Abnormal } & \multicolumn{3}{|c|}{ Training cohort $(n=300)$} & \multicolumn{3}{|c|}{$\begin{array}{l}\text { Validation cohort } 1 \\
(n=143)\end{array}$} \\
\hline & $6(8.6)$ & $6(8.1)$ & & $4(9.5)$ & $6(6.6)$ & \\
\hline TG (\%) & & & 0.095 & & & 0.335 \\
\hline Normal & $44(62.9)$ & $56(75.7)$ & & $27(64.3)$ & $66(72.5)$ & \\
\hline Abnormal & $26(37.1)$ & $18(24.3)$ & & $15(35.7)$ & $25(27.5)$ & \\
\hline TGAB (\%) & & & 0.143 & & & 0.596 \\
\hline Normal & $40(57.1)$ & $51(68.9)$ & & $32(76.2)$ & $73(80.2)$ & \\
\hline Abnormal & $30(42.9)$ & $23(31.1)$ & & $10(23.8)$ & $18(19.8)$ & \\
\hline TPOAB (\%) & & & 0.341 & & & 0.863 \\
\hline Normal & $48(68.6)$ & $56(75.7)$ & & $36(85.7)$ & $79(86.8)$ & \\
\hline Abnormal & $22(31.4)$ & $18(24.3)$ & & $6(14.3)$ & $12(13.2)$ & \\
\hline Tumor size (mm) & & & $<0.001$ & & & $<0.001$ \\
\hline Mean \pm SD & $14.9 \pm 7.1$ & $8.3 \pm 7.0$ & & $\begin{array}{l}14.0 \pm \\
11.2\end{array}$ & $8.3 \pm 7.9$ & \\
\hline Tumor size $(\mathrm{mm})$ & & & $<0.001$ & & & 0.001 \\
\hline$\geq 1$ & $41(58.6)$ & $20(27.0)$ & & $33(78.6)$ & $20(22.0)$ & \\
\hline$<1$ & $29(41.4)$ & $54(73.0)$ & & $9(21.4)$ & $71(78.0)$ & \\
\hline $\begin{array}{l}\text { US-reported central } \\
\text { CLN status (\%) }\end{array}$ & & & $<0.001$ & & & $<0.001$ \\
\hline negative & $38(54.3)$ & $68(91.9)$ & & $20(47.6)$ & $78(85.7)$ & \\
\hline positive & $32(45.7)$ & $6(8.1)$ & & $22(52.4)$ & $13(14.3)$ & \\
\hline Radiomics score & & & $<0.001$ & & & $<0.001$ \\
\hline Median & -0.2431 & -0.4058 & & -0.2695 & -0.4438 & \\
\hline (interquartile range) & $\begin{array}{l}(-0.3553 \\
-0.1270)\end{array}$ & $\begin{array}{l}(-0.5119 \\
-0.3104)\end{array}$ & & $\begin{array}{l}(-0.3652 \\
-0.1542)\end{array}$ & $\begin{array}{l}(-0.5602 \\
-0.3217)\end{array}$ & \\
\hline $\begin{array}{l}\text { CLN, cervical lymph } n \\
\text { antibody; TPOAB, thy } \\
\text { metastasis; SD, stand } \\
\text { boldface. }\end{array}$ & $\begin{array}{l}\text { CLNM, cer } \\
\text { eroxidase } \\
\text { eviation; } \iota\end{array}$ & $\begin{array}{l}\text { lymph me } \\
\text { oody; TSH } \\
\text { trasound. }\end{array}$ & $\begin{array}{l}\text { tasis; TG, } t \\
\text { /roid stimu } \\
\text { nificant dif }\end{array}$ & $\begin{array}{l}\text { nyroglobulir } \\
\text { ating horm } \\
\text { ferences are }\end{array}$ & $\begin{array}{l}\text { TGAB, thyrog } \\
\text { e; LNM, lymp } \\
\text { ighlighted in }\end{array}$ & $\begin{array}{l}\text { bulin } \\
\text { node }\end{array}$ \\
\hline
\end{tabular}


Table 2

Associations between the lateral CLN status and clinical parameters in the training and validation cohorts Training cohort $(n=300)$

Validation cohort 1 $(n=143)$

\begin{tabular}{|c|c|c|c|c|c|c|}
\hline Characteristic & $\begin{array}{l}\text { LLNM + } \\
(n=27)\end{array}$ & $\begin{array}{l}\text { LLNM - } \\
(\mathrm{n}=273)\end{array}$ & $p$ & $\begin{array}{l}\text { LLNM + } \\
(n=15)\end{array}$ & $\begin{array}{l}\text { LLNM - } \\
(n=128)\end{array}$ & $p$ \\
\hline Age (years) & & & 0.515 & & & 0.543 \\
\hline Mean \pm SD & $40.1 \pm 10.9$ & $42.6 \pm 12.0$ & & $\begin{array}{l}41.5 \pm \\
16.9\end{array}$ & $\begin{array}{l}43.5 \pm \\
11.0\end{array}$ & \\
\hline Age (years) & & & 0.221 & & & 0.440 \\
\hline$>45$ & $8(29.6)$ & $114(41.8)$ & & $5(33.3)$ & $56(43.8)$ & \\
\hline$\leq 45$ & $19(70.4)$ & $159(58.2)$ & & $10(66.7)$ & $72(56.2)$ & \\
\hline Gender (\%) & & & 0.159 & & & 0.542 \\
\hline Male & $11(40.7)$ & $76(27.8)$ & & $3(20.0)$ & $35(27.3)$ & \\
\hline Female & $16(59.3)$ & $197(72.2)$ & & $12(80.0)$ & 93 (72.7) & \\
\hline TSH (\%) & & & 0.762 & & & 0.950 \\
\hline Normal & $25(92.6)$ & $248(90.8)$ & & $14(93.3)$ & $120(93.8)$ & \\
\hline Abnormal & $2(7.4)$ & $25(9.2)$ & & $1(6.7)$ & $8(6.3)$ & \\
\hline TG (\%) & & & 0.001 & & & 0.054 \\
\hline Normal & $20(74.1)$ & $255(93.4)$ & & $12(80.0)$ & $122(95.3)$ & \\
\hline Abnormal & $7(25.9)$ & $18(6.6)$ & & $3(20.0)$ & $6(4.7)$ & \\
\hline TGAB (\%) & & & 0.338 & & & 0.984 \\
\hline Normal & $15(55.6)$ & $177(64.8)$ & & $10(66.7)$ & $85(66.4)$ & \\
\hline Abnormal & $12(44.4)$ & $96(35.2)$ & & $5(33.3)$ & $43(33.6)$ & \\
\hline TPOAB (\%) & & & 0.254 & & & 0.847 \\
\hline Normal & $17(63.0)$ & $200(73.3)$ & & $12(80.0)$ & $105(82.0)$ & \\
\hline Abnormal & $10(37.0)$ & $73(26.7)$ & & $3(20.0)$ & $23(18.0)$ & \\
\hline Tumor size (mm) & & & $<0.001$ & & & $<0.001$ \\
\hline
\end{tabular}

CLN, cervical lymph node; CLNM, cervical lymph metastasis; TG, thyroglobulin; TGAB, thyroglobulin antibody; TPOAB, thyroid peroxidase antibody; TSH, thyroid stimulating hormone; LNM, lymph node metastasis; SD, standard deviation; US, ultrasound. Significant differences are highlighted in boldface. 


\begin{tabular}{|c|c|c|c|c|c|c|}
\hline \multirow[b]{2}{*}{ Mean \pm SD } & \multicolumn{3}{|c|}{ Training cohort $(n=300)$} & \multicolumn{3}{|c|}{$\begin{array}{l}\text { Validation cohort } 1 \\
(n=143)\end{array}$} \\
\hline & $15.1 \pm 6.9$ & $9.0 \pm 6.8$ & & $\begin{array}{l}16.1 \pm \\
11.7\end{array}$ & $8.8 \pm 6.7$ & \\
\hline Tumor size $(\mathrm{mm})$ & & & $<0.001$ & & & $<0.001$ \\
\hline$\geq 1$ & $23(85.2)$ & $94(34.4)$ & & $11(73.3)$ & $33(25.8)$ & \\
\hline$<1$ & $4(14.8)$ & $179(65.6)$ & & $4(26.7)$ & $95(74.2)$ & \\
\hline $\begin{array}{l}\text { US-reported lateral } \\
\text { CLN status (\%) }\end{array}$ & & & $<0.001$ & & & $<0.001$ \\
\hline negative & $7(25.9)$ & $243(89.0)$ & & $4(26.7)$ & $116(90.6)$ & \\
\hline positive & $20(74.1)$ & $30(11.0)$ & & $11(73.3)$ & $12(9.4)$ & \\
\hline $\begin{array}{l}\text { Radiomics score } \\
\text { Mean }\end{array}$ & -0.0318 & -0.1346 & $<0.001$ & -0.0590 & -0.1414 & $<0.001$ \\
\hline \multirow[t]{2}{*}{ (interquartile range) } & $\begin{array}{l}(-0.1413 \\
-0.0081)\end{array}$ & $\begin{array}{l}(-0.1706 \\
-0.1114)\end{array}$ & & $\begin{array}{l}(-0.1357 \\
-0.0323)\end{array}$ & $\begin{array}{l}(-0.1973- \\
0.0986)\end{array}$ & \\
\hline & \multicolumn{3}{|c|}{$\begin{array}{l}\text { Validation cohort } 2 \\
(\mathrm{n}=144)\end{array}$} & \multicolumn{3}{|c|}{$\begin{array}{l}\text { Validation cohort } 3 \\
(n=133)\end{array}$} \\
\hline Characteristic & $\begin{array}{l}\operatorname{LLNM}+ \\
(n=15)\end{array}$ & $\begin{array}{l}\text { LLNM - } \\
(n=129)\end{array}$ & $p$ & $\begin{array}{l}\text { LLNM + } \\
(n=19)\end{array}$ & $\begin{array}{l}\text { LLNM - } \\
(n=114)\end{array}$ & $p$ \\
\hline Age (years) & & & 0.992 & & & 0.007 \\
\hline Mean \pm SD & $39.7 \pm 14.1$ & $39.7 \pm 11.4$ & & $\begin{array}{l}38.8 \pm \\
10.7\end{array}$ & $\begin{array}{l}44.3 \pm \\
12.3\end{array}$ & \\
\hline Age (years) & & & 0.553 & & & 0.521 \\
\hline$>45$ & $7(46.7)$ & $50(38.8)$ & & $7(36.8)$ & $51(44.7)$ & \\
\hline$\leq 45$ & $8(53.3)$ & $79(61.2)$ & & $12(63.2)$ & $63(55.3)$ & \\
\hline Gender (\%) & & & 0.270 & & & 0.816 \\
\hline Male & $2(13.3)$ & $34(26.4)$ & & $6(28.9)$ & $33(31.6)$ & \\
\hline Female & $13(86.7)$ & $95(73.6)$ & & $13(71.1)$ & $81(68.4)$ & \\
\hline TSH (\%) & & & 0.459 & & & 0.154 \\
\hline Normal & $13(86.7)$ & $119(92.2)$ & & $16(84.2)$ & $107(93.9)$ & \\
\hline
\end{tabular}

CLN, cervical lymph node; CLNM, cervical lymph metastasis; TG, thyroglobulin; TGAB, thyroglobulin antibody; TPOAB, thyroid peroxidase antibody; TSH, thyroid stimulating hormone; LNM, lymph node metastasis; SD, standard deviation; US, ultrasound. Significant differences are highlighted in boldface. 


\begin{tabular}{|c|c|c|c|c|c|c|}
\hline \multirow[b]{2}{*}{ Abnormal } & \multicolumn{3}{|c|}{ Training cohort $(n=300)$} & \multicolumn{3}{|c|}{$\begin{array}{l}\text { Validation cohort } 1 \\
(n=143)\end{array}$} \\
\hline & $2(13.3)$ & $10(7.8)$ & & $3(15.8)$ & $7(6.1)$ & \\
\hline TG (\%) & & & 0.730 & & & 0.487 \\
\hline Normal & $11(73.3)$ & $89(69.0)$ & & $12(63.2)$ & $81(71.1)$ & \\
\hline Abnormal & $4(26.7)$ & $40(31.0)$ & & $7(36.8)$ & $33(28.9)$ & \\
\hline TGAB (\%) & & & 0.161 & & & 0.224 \\
\hline Normal & $7(46.7)$ & $84(65.1)$ & & $17(89.5)$ & $88(77.2)$ & \\
\hline Abnormal & $8(53.3)$ & $45(34.9)$ & & $2(10.5)$ & $26(22.8)$ & \\
\hline TPOAB (\%) & & & 0.084 & & & 0.679 \\
\hline Normal & $8(53.3)$ & $96(74.4)$ & & $17(89.5)$ & $98(86.0)$ & \\
\hline Abnormal & $7(46.7)$ & $33(25.6)$ & & $2(10.5)$ & $16(14.0)$ & \\
\hline Tumor size $(\mathrm{mm})$ & & & $<0.001$ & & & $<0.001$ \\
\hline Mean \pm SD & $14.9 \pm 7.1$ & $8.3 \pm 7.0$ & & $15.7 \pm 7.2$ & $8.5 \pm 6.9$ & \\
\hline Tumor size $(\mathrm{mm})$ & & & 0.002 & & & 0.001 \\
\hline$\geq 1$ & $12(80.0)$ & $49(38.0)$ & & $14(73.7)$ & $39(34.2)$ & \\
\hline$<1$ & $3(20.0)$ & $80(62.0)$ & & $5(26.3)$ & $75(65.8)$ & \\
\hline $\begin{array}{l}\text { US-reported lateral } \\
\text { CLN status (\%) }\end{array}$ & & & $<0.001$ & & & $<0.001$ \\
\hline negative & $4(26.7)$ & $112(86.8)$ & & $5(26.3)$ & $99(86.8)$ & \\
\hline positive & $11(73.3)$ & $17(13.2)$ & & $14(73.7)$ & 15 (13.2) & \\
\hline Radiomics score & & & $<0.001$ & & & $<0.001$ \\
\hline Mean & -0.0629 & -0.1501 & & -0.0496 & -0.1345 & \\
\hline (interquartile range) & $\begin{array}{l}(-0.1347 \\
-0.0033)\end{array}$ & $\begin{array}{l}(-0.2014 \\
-0.1020)\end{array}$ & & $\begin{array}{l}(-0.1300 \\
-0.0029)\end{array}$ & $\begin{array}{l}(-0.1798 \\
-0.1008)\end{array}$ & \\
\hline $\begin{array}{l}\text { CLN, cervical lymph } n \\
\text { antibody; TPOAB, thy } \\
\text { metastasis; SD, stand } \\
\text { boldface. }\end{array}$ & $\begin{array}{l}\text { CLNM, cer } \\
\text { peroxidase } \\
\text { deviation; U }\end{array}$ & $\begin{array}{l}\text { al lymph me } \\
\text { tibody; TSH, } \\
\text { ultrasound. }\end{array}$ & $\begin{array}{l}\text { asis; TG } \\
\text { roid stim } \\
\text { ificant c }\end{array}$ & $\begin{array}{l}\text { hyroglobulin } \\
\text { lating hormc } \\
\text { ferences are }\end{array}$ & $\begin{array}{l}\text { TGAB, thyrog } \\
\text { e; LNM, lym } \\
\text { ighlighted in }\end{array}$ & $\begin{array}{l}\text { bulin } \\
\text { node }\end{array}$ \\
\hline
\end{tabular}

\section{Feature Selection And Development Of The Us Radiomics Signatures}


The procedure of feature extraction has been described in detail(16). Briefly, in the training set, 614 reproducible features were extracted from the original thyroid US images. Excellent intra- and interobserver reproducibility of feature extraction was achieved, with intra-observer ICCs ranging from 0.823 to 0.932 , and the inter-observer ICCs ranging from 0.841 to 0.954 . The detailed procedures of the feature selection and the radiomics signature construction for predicting the central and lateral cervical LNM were described in our previous studies $(15,20)$, respectively.

\section{Validation Of The Radiomics Signatures}

The Rad-score were significantly higher in the central and lateral LNM groups in both the training set and the validation sets than that in the non-LNM groups $(p<0.001, p<0.001$, Table 1 and 2$)$. The ROC curves were shown in figure 1 and 2 . The radiomics signature yielded an AUC of 0.839 for discriminating between central/non-central LNM groups in the training set, and $0.819,0.799,0.797$, respectively, in the validation sets 1,2 , and 3 . The AUC for differentiating between lateral/non-lateral LNM groups was 0.908 in the training set, and were $0.888,0.796,0.793$, respectively, in the validation cohorts 1,2 , and 3 . The radiomics signatures yielded a better discriminatory performance than that of the US-reported LN status (Table 3 and 4). 
Table 3

Diagnostic performance of US, radiomics signature, and nomogram in the training and validation cohorts

\section{Cohorts}

Sensitivity

(\%) US-reported central LN status in training
cohort

Radiomics signature in training cohort

Radiomics nomogram in training cohort

US-reported central LN status in

validation cohort 1

Radiomics signature in validation cohort $\quad 78.7$

1

Radiomics nomogram in validation cohort 1

US-reported central LN status in validation cohort 2

Radiomics signature in validation cohort 2

Radiomics nomogram in validation cohort 2

US-reported central LN status in validation cohort 3

Radiomics signature in validation cohort $\quad 71.4$ 3

Radiomics nomogram in validation cohort 3

50.0

83.7

83.7

48.9

83.0

45.7

65.7

77.1

52.4

71.4
US, ultrasound; $\mathrm{CT}$, computed tomography; $\mathrm{AUC}$, area under curve; $\mathrm{Cl}$, confidence interval.
Specificity AUC $(95 \% \mathrm{Cl})$

(\%)

Accuracy

(\%)

76.3

$0.768)$

81.1

0.839 (0.792-

0.887 )

81.6

0.875 (0.834-

$0.915)$

90.6

0.698 (0.599-

$0.797)$

81.2

0.819 (0.738-

0.900 )

82.3

0.857 (0.785-

0.929)

82.0

82.3

76.9

80.4

91.9

0.688 (0.600-

0.776 )

79.7

0.799 (0.729-

0.869 )

85.1

0.880 (0.826-

$0.934)$

85.7

0.690 (0.587-

0.794 )

74.7

0.797 (0.716-

0.879)

87.9

0.870 (0.808-

0.932 )
82.5

69.4

72.9

81.3

75.1

73.7

82.7 
Table 4

Diagnostic performance of US, radiomics signature, and nomogram in the training and validation cohorts

\begin{tabular}{|c|c|c|c|c|}
\hline Cohorts & $\begin{array}{l}\text { Sensitivity } \\
\text { (\%) }\end{array}$ & $\begin{array}{l}\text { Specificity } \\
(\%)\end{array}$ & AUC $(95 \% \mathrm{Cl})$ & $\begin{array}{l}\text { Accuracy } \\
(\%)\end{array}$ \\
\hline $\begin{array}{l}\text { US-reported lateral LN status in training } \\
\text { cohort }\end{array}$ & 74.1 & 89.0 & $\begin{array}{l}0.815(0.716- \\
0.915)\end{array}$ & 87.6 \\
\hline Radiomics signature in training cohort & 77.8 & 90.1 & $\begin{array}{l}0.908(0.845- \\
0.972)\end{array}$ & 89.0 \\
\hline Radiomics nomogram in training cohort & 81.5 & 94.1 & $\begin{array}{l}0.938(0.887- \\
0.989)\end{array}$ & 93.0 \\
\hline $\begin{array}{l}\text { US-reported lateral LN status in } \\
\text { validation cohort }\end{array}$ & 73.3 & 90.6 & $\begin{array}{l}0.820(0.684- \\
0.955)\end{array}$ & 88.8 \\
\hline $\begin{array}{l}\text { Radiomics signature in validation cohort } \\
1\end{array}$ & 80.0 & 91.4 & $\begin{array}{l}0.888(0.786- \\
0.990)\end{array}$ & 90.2 \\
\hline $\begin{array}{l}\text { Radiomics nomogram in validation } \\
\text { cohort } 1\end{array}$ & 86.7 & 92.2 & $\begin{array}{l}0.905(0.789- \\
0.999)\end{array}$ & 91.6 \\
\hline $\begin{array}{l}\text { US-reported lateral LN status in } \\
\text { validation cohort } 2\end{array}$ & 73.3 & 86.8 & $\begin{array}{l}0.801(0.665- \\
0.936)\end{array}$ & 85.4 \\
\hline $\begin{array}{l}\text { Radiomics signature in validation cohort } \\
2\end{array}$ & 66.7 & 82.9 & $\begin{array}{l}0.796(0.684- \\
0.909)\end{array}$ & 84.7 \\
\hline $\begin{array}{l}\text { Radiomics nomogram in validation } \\
\text { cohort } 2\end{array}$ & 73.3 & 93.0 & $\begin{array}{l}0.884(0.785- \\
0.984)\end{array}$ & 90.9 \\
\hline $\begin{array}{l}\text { US-reported lateral LN status in } \\
\text { validation cohort } 3\end{array}$ & 73.7 & 86.8 & $\begin{array}{l}0.803(0.681- \\
0.924)\end{array}$ & 85.0 \\
\hline $\begin{array}{l}\text { Radiomics signature in validation cohort } \\
3\end{array}$ & 68.4 & 75.4 & $\begin{array}{l}0.793(0.683- \\
0.903)\end{array}$ & 74.4 \\
\hline $\begin{array}{l}\text { Radiomics nomogram in validation } \\
\text { cohort } 3\end{array}$ & 78.9 & 89.5 & $\begin{array}{l}0.906(0.841- \\
0.971)\end{array}$ & 88.0 \\
\hline
\end{tabular}

\section{Prediction Of Ln Status Based On Radiomics Nomogram}

The age (<45), US-reported central CLN status, and radiomics signature were identified as independent predictive factors for central LNM; the US-reported lateral CLN status and radiomics signature were identified as independent variables for lateral LNM by multivariate logistic regression analysis (Table 5 and 6). The two radiomics nomograms were constructed by incorporating the independent predictors, respectively (Figure 3 ). In the training sets, the radiomics nomogram showed the highest discrimination between central LNM positive and negative with an AUC of $0.875(95 \% \mathrm{Cl}, 0.834-0.915)$; the nomogram for discriminating between the lateral LNM positive and negative also yielded the greatest AUC $(0.938$, 
95\% Cl: 0.887-0.989), which indicated that nomogram achieved better discriminatory performance than either the US-reported CLN status or the radiomics signature. The favorable discrimination was also observed in the validation cohorts (Table 3 and 4). Thus, our nomogram performed well in both the training cohort and the validation cohorts. The calibration curve of the radiomics nomograms for the probability of central/lateral LNM demonstrated an optimal consistency between prediction and pathologic observation in the training and validation cohorts (Figure 4 and 5). The Hosmer-Lemeshow test showed no statistical significance, which suggested no significant deviation from a perfect fit.

Table 5

Independent predictive factors for central LNM in the radiomics nomogram

\begin{tabular}{|llll|}
\hline Variables & $\boldsymbol{\beta}$ & Odds Ratio (95\% Cl) & $\boldsymbol{p}$ \\
\hline Age & 0.934 & $2.544(1.317-4.913)$ & 0.005 \\
\hline US-reported central CLN status & 2.007 & $7.444(3.597-15.406)$ & $<0.001$ \\
\hline US radiomics signature & 6.354 & $574.537(96.796-3410.181)$ & $<0.001$ \\
\hline Constant & 0.473 & & 0.225 \\
\hline Cl, confidence interval; CLN indicates cervical lymph nodes; US, ultrasound. \\
\hline
\end{tabular}

Table 6

Independent predictive factors for lateral LNM in the radiomics nomogram

\begin{tabular}{|llll|}
\hline Variables & $\boldsymbol{\beta}$ & Odds Ratio (95\% Cl) & $\boldsymbol{p}$ \\
\hline US-reported central CLN status & 25.752 & $142.012(28.751-701.016)$ & $<0.001$ \\
\hline US radiomics signature & 2.693 & $14.772(4.312-50.607)$ & $<0.001$ \\
\hline Constant & -1.125 & & 0.031 \\
\hline Cl, confidence interval; CLN indicates cervical lymph nodes; US, ultrasound. \\
\hline
\end{tabular}

\section{Clinical Significance}

The decision curve analyses (DCA) for the US-reported CLN status, the radiomics signature, and the nomograms are illustrated in figure 6 . The DCA demonstrated that the nomogram exhibited an optimal net benefit to predict the central LNM than other models for threshold probability within the range of 0 0.98 , and the prediction of lateral LNM with radiomics nomogram could benefit more as compared to the radiomics signature and the US-reported lateral CLN status when the threshold probability ranged from 0.04 to 0.98 .

\section{Discussion}


In this study, we developed and validated two radiomics nomograms to improve the accuracy in preoperatively predicting the central and lateral LNM, respectively, in a non-invasive and individualized fashion. In this multi-institutional study, the two nomograms showed favorable predictive ability in both training and independent validation cohorts, outperforming the radiologists' subjective prediction of cervical LNM and the radiomics signature. Furthermore, the DCA also demonstrated that the nomograms had the best net benefit within a wide range of threshold probabilities. These results highlighted that our radiomics nomograms provided a new method for the preoperatively individualized prediction of central and lateral LNM in PTC patients. This approach might greatly facilitate the decision-making in the clinical practice.

Accurate preoperative predicting the cervical LNM of patients with PTC is of great importance for guiding clinical treatment, particularly, for surgeons to determine the extent of the surgical resection and assessing the necessity of CLN dissection $(3,13)$. Neck US examination plays a pivotal role in PTC staging. Unfortunately, the detection rate of cervical LNM is not desirable. Since US is unbale to consistently visualize the deep anatomic structures, or structures which are acoustically shadowed by air and bone(22), in many patients, the LNM in the central compartment may not show any abnormal finding in preoperative US examination(23). Besides, US examination is an empirical diagnosis which is greatly affected by the expertise of the operator, therefore, prone to interobserver variability when determining lateral LNM(8). Many studies have investigated the association between cervical LNM and the morphologic US features of primary tumor, and have reported that the "taller than wide" shape, tumor size, presence of calcification, and closer distance between tumor and capsule were independent risk factors for $\operatorname{LNM}(15,24-26)$. Although, the US features mentioned above are encouraging, the imaging features are also based on the judgement of the performing physician and thus, lack objectivity. However, utilizing imaging features of the primary tumor enables an approach which is less affected by the expertise of the operator, thus could be a promising tool for clinical practice(8).

Radiomics is a novel method, which extracts high-throughput quantitative information from standard-ofcare medical imaging that improves medical decision-making, has drawn increased attention in cancer research $(27,28)$. Currently, radiomics has been explored in the field of thyroid carcinoma research, such as in the differential diagnosis of thyroid nodules(29), prediction of the cervical LNM(16), extrathyroidal extension(30), BRAF ${ }^{\mathrm{V} 600 \mathrm{E}}$ mutation(31), as well as the survival prediction(32). Lu et al. developed a nomogram based on the contrast-enhanced CT, which showed favorable performance in predicting LNM in PTC patients with an AUC of 0.867(33). Nevertheless, iodinated contrast agents have a risk of contrast allergy and might delay the RAI in PTC patients, which limits its clinical application. Hu et al. established an MRI radiomics model which obtained good accuracy in predicting LNM status preoperatively(6). However, MRI is expensive, time-consuming, and not routinely performed for PTC patients in clinical management.

Our previous study has demonstrated the feasibility of applying the US-based radiomics analysis for assessing the risk of LNM in PTC patients(16). Jiang et al. established a nomogram which combined the shear-wave elastography (SWE) radiomics signature and clinicopathological parameters achieved

Page $17 / 25$ 
satisfactory predictive value for LN staging in PTC patients, with an AUC of 0.851 and 0.832 in the training and validation cohort, respectively(23). These US radiomics studies were designed to predict the cervical LNM, however, the treatment strategy for LNM in the central or lateral compartment vary greatly. Thus, accurate radiomics models for predicting LNM in central or lateral compartment, respectively, are urgently needed.

Here, we attempted to develop two US radiomics nomograms for predicting the central and/or lateral LNM preoperatively. We investigated the association between central/ lateral LNM and preoperatively available characteristics by univariate analysis. Age, US-reported central CLN status, and radiomics signature were identified as the independent predictors for central LNM; US-reported lateral CLN status and radiomics signature were identified as the independent risk factors for lateral LNM, respectively, by multivariate logistic regression analyses. Then, two nomograms were developed based on the multivariate analyses. Interestingly, the independent risk factors on which the two nomograms were based in this study were also the independent risk factors for central/lateral LNM in our previous studies $(15,20)$. This may indicate the great predictive value of these factors for cervical LNM in PTC patients. The nomograms have been confirmed to be capable of generating a probability of central/lateral LNM preoperatively and individually, which is in line with the prevailing concept of personalized precision medicine. The calibration curve showed superior consistency between nomogramestimated and actual observed probability in each of the cohort. For the clinical usefulness of the nomograms, we employed DCA to evaluate whether the nomogram-assisted decision-making would improve the patient outcomes. Our results demonstrated that the nomogram supplied better clinical net benefit across the majority range of reasonable threshold probabilities than either the radiomics signature or the US-reported CLN status.

Some limitations have to be acknowledged in this study. First, the gene mutation status was not included in this study. Recently, increasing studies have been conducted to investigate the association between gene mutation and the LNM in PTC. Since not all the patients received the BRAF mutation examination after FNA, the role of gene mutation as an independent predictor still needs to be further studied. Second, the radiomics features were only extracted from conventional US images. However, multimodal US technology, such as SWE or contrast-enhanced US, has been applied jointly for the diagnosis of thyroid nodules in clinical practice, a new radiomics study incorporating multimodal technology is ongoing at our center. Third, the utilized images were obtained from the same US system. Radiomics features have been reported to be affected by the US machine and parameters used for image acquisition, thus, a multicenter study with multiple US systems is needed to acquire high-level evidence for further clinical application.

In conclusion, we developed an easy-to-use and noninvasive predictive tool which incorporates the radiomics signature and clinical characteristics to preoperatively evaluate the individual risk of cervical central/lateral LNM in patients with PTC. The nomograms proposed here hold promise for optimizing the personalized treatment, which may improve the survival outcome in PTC patients. 


\section{Abbreviation List}

\begin{tabular}{|ll|}
\hline LNM & Lymph node metastasis \\
\hline PTC & Papillary thyroid carcinoma \\
\hline CLN & Ultrasound \\
\hline LN & Cervical lymph node \\
\hline CT & Lymph node \\
\hline FNA & Computed tomography \\
\hline CCND & Central compartment neck dissection \\
\hline RAI & Radioactive iodine \\
\hline Rad-score & Radiomics score \\
\hline AUC & Area under the ROC curve \\
\hline TSH & Thyroid stimulating hormone \\
\hline TG & Thyroglobulin \\
\hline TPOAB & Thyroglobulin antibody \\
\hline ICC & Interclass correlation coefficient \\
\hline LASSO & Least absolute shrinkage and selection operator \\
\hline ROC & Receiver operating characteristic \\
\hline DCA & Decision curve analysis \\
\hline SD & Standard deviation \\
\hline SWE & Shear-wave elastography \\
\hline
\end{tabular}

\section{Declarations}

\section{Ethics approval and consent to participate}

The authors are accountable for all aspects of the work in ensuring that questions related to the accuracy or integrity of any part of the work are appropriately investigated and resolved. All data included in the present study was collected after obtaining ethical approval from the institutional review boards at Fudan University Shanghai Cancer Center, Fudan University Shanghai Cancer Center, Ruijin Hospital, School of Medicine, Shanghai Jiao Tong University, and Zhongshan Hospital, Fudan University (No. 1802181-22NSFC). This retrospective study was approved by the Ethics Committees of all participating hospitals 
(Fudan University Shanghai Cancer Center; Ruijin Hospital, School of Medicine, Shanghai Jiao Tong University; Zhongshan Hospital, Fudan University), and performed in accordance with the Declaration of Helsinki. The need for informed consent was waived by the Institutional Review Boards of Fudan University Shanghai Cancer Center, Ruijin Hospital, School of Medicine, Shanghai Jiao Tong University; Zhongshan Hospital, Fudan University, because of the retrospective nature of the study.

\section{Consent for publication}

Not applicable.

\section{Availability of data and materials}

The datasets used and analyzed during the current study are available from the corresponding author on reasonable request.

\section{Competing interests}

The authors declare that they have no competing interests.

\section{Funding}

This work was supported by the National Natural Science Foundation of China (82071945), the Science and Technology Commission of Shanghai Municipality (17411963300).

\section{Author Contribution}

(I) Conception and design: Yuyang Tong; Jingwen Zhang

(II) Administrative support: Shichong Zhou; Hansheng Xia

(III) Provision of study materials or patients: Yuyang Tong; Jingwen Zhang

(IV) Collection and assembly of data: Yi Wei; Jinhua Yu; Weiwei Zhan; Yuanyuan Wang; Cai Chang

(V) Data analysis and interpretation: Yuyang Tong; Jingwen Zhang

(VI) Manuscript writing: All authors

(VII) Final approval of manuscript: All authors

\section{Acknowledgement}

We thank Dr. Zezhou Wang for his specialized consultation on the statistical analyses. Yuyang Tong and Jingwen Zhang contributed equally to the manuscript. This work was supported by the National Natural Science Foundation of China (82071945), the Science and Technology Commission of Shanghai Municipality (17411963300). 


\section{References}

1. Sung H, Ferlay J, Siegel RL, Laversanne M, Soerjomataram I, Jemal A, et al. Global Cancer Statistics 2020: GLOBOCAN Estimates of Incidence and Mortality Worldwide for 36 Cancers in 185 Countries. CA Cancer J Clin. 2021;71:209-49.

2. Huang C, Cong S, Liang T, Feng Z, Gan K, Zhou R, et al. Development and validation of an ultrasoundbased nomogram for preoperative prediction of cervical central lymph node metastasis in papillary thyroid carcinoma. Gland Surg. 2020;9:956-67.

3. Li T, Li H, Xue J, Miao J, Kang C. Shear wave elastography combined with gray-scale ultrasound for predicting central lymph node metastasis of papillary thyroid carcinoma. Surg Oncol. 2021;36:1-6.

4. Chen L, Chen L, Liu J, Wang B, Zhang H. Value of Qualitative and Quantitative Contrast-Enhanced Ultrasound Analysis in Preoperative Diagnosis of Cervical Lymph Node Metastasis From Papillary Thyroid Carcinoma. J Ultrasound Med. 2020;39:73-81.

5. Liang K, He L, Dong W, Zhang H. Risk factors of central lymph node metastasis in cNO papillary thyroid carcinoma: a study of 529 patients. Med Sci Monit. 2014;20:807-11.

6. Hu W, Wang H, Wei R, Wang L, Dai Z, Duan S, et al. MRI-based radiomics analysis to predict preoperative lymph node metastasis in papillary thyroid carcinoma. Gland Surg. 2020;9:1214-26.

7. Haugen BR, Alexander EK, Bible KC, Doherty GM, Mandel SJ, Nikiforov YE, et al. 2015 American Thyroid Association Management Guidelines for Adult Patients with Thyroid Nodules and Differentiated Thyroid Cancer: The American Thyroid Association Guidelines Task Force on Thyroid Nodules and Differentiated Thyroid Cancer. Thyroid. 2016;26:1-133.

8. Park VY, Han K, Kim HJ, Lee E, Youk JH, Kim EK, et al. Radiomics signature for prediction of lateral lymph node metastasis in conventional papillary thyroid carcinoma. PLoS One. 2020;15:e0227315.

9. Gao L, Wang J, Jiang Y, Gao Q, Wang Y, Xi X, et al. The Number of Central Lymph Nodes on Preoperative Ultrasound Predicts Central Neck Lymph Node Metastasis in Papillary Thyroid Carcinoma: A Prospective Cohort Study. Int J Endocrinol. 2020;2020:2698659.

10. Wang H, Song B, Ye N, Ren J, Sun X, Dai Z, et al. Machine learning-based multiparametric MRI radiomics for predicting the aggressiveness of papillary thyroid carcinoma. Eur $\mathrm{J}$ Radiol. 2020;122:108755.

11. Moon HJ, Kim EK, Yoon JH, Kwak JY. Differences in the diagnostic performances of staging US for thyroid malignancy according to experience. Ultrasound Med Biol. 2012;38:568-73.

12. Liu Y, Zhou H, Yang P, Zhou Y, Wu J, Chen C, et al. Contrast-enhanced ultrasonography features of papillary thyroid carcinoma for predicting cervical lymph node metastasis. Exp Ther Med. 2017;14:4321-7.

13. Zhang H, Hu S, Wang X, He J, Liu W, Yu C, et al. Prediction of Cervical Lymph Node Metastasis Using MRI Radiomics Approach in Papillary Thyroid Carcinoma: A Feasibility Study. Technol Cancer Res Treat. 2020;19:1533033820969451. 
14. Limkin EJ, Sun R, Dercle L, Zacharaki El, Robert C, Reuzé S, et al. Promises and challenges for the implementation of computational medical imaging (radiomics) in oncology. Ann Oncol. 2017;28:1191-206.

15. Tong Y, Li J, Huang Y, Zhou J, Liu T, Guo Y, et al. Ultrasound-Based Radiomic Nomogram for Predicting Lateral Cervical Lymph Node Metastasis in Papillary Thyroid Carcinoma. Acad Radiol. 2021;28:1675-84.

16. Liu T, Zhou S, Yu J, Guo Y, Wang Y, Zhou J, et al. Prediction of Lymph Node Metastasis in Patients With Papillary Thyroid Carcinoma: A Radiomics Method Based on Preoperative Ultrasound Images. Technol Cancer Res Treat. 2019;18:1533033819831713.

17. Yu J, Deng Y, Liu T, Zhou J, Jia X, Xiao T, et al. Lymph node metastasis prediction of papillary thyroid carcinoma based on transfer learning radiomics. Nat Commun. 2020;11:4807.

18. Kim SS, Lee BJ, Lee JC, Song SH, Kim BH, Son SM, et al. Preoperative serum thyroid stimulating hormone levels in well-differentiated thyroid carcinoma is a predictive factor for lateral lymph node metastasis as well as extrathyroidal extension in Korean patients: a single-center experience. Endocrine. 2011;39:259-65.

19. Shen CT, Zhang XY, Qiu ZL, Sun ZK, Wei WJ, Song HJ, et al. Thyroid autoimmune antibodies in patients with papillary thyroid carcinoma: a double-edged sword? Endocrine. 2017;58:176-83.

20. Zhou SC, Liu TT, Zhou J, Huang YX, Guo Y, Yu JH, et al. An Ultrasound Radiomics Nomogram for Preoperative Prediction of Central Neck Lymph Node Metastasis in Papillary Thyroid Carcinoma. Front Oncol. 2020;10:1591.

21. Tessler FN, Middleton WD, Grant EG, Hoang JK, Berland LL, Teefey SA, et al. ACR Thyroid Imaging, Reporting and Data System (TI-RADS): White Paper of the ACR TI-RADS Committee. J Am Coll Radiol. 2017;14:587-595.

22. Guo L, Ma YQ, Yao Y, Wu M, Deng ZH, Zhu FW, et al. Role of ultrasonographic features and quantified BRAFV600E mutation in lymph node metastasis in Chinese patients with papillary thyroid carcinoma. Sci Rep. 2019;9:75.

23. Jiang M, Li C, Tang S, Lv W, Yi A, Wang B, et al. Nomogram Based on Shear-Wave Elastography Radiomics Can Improve Preoperative Cervical Lymph Node Staging for Papillary Thyroid Carcinoma. Thyroid. 2020;30:885-97.

24. Zhan J, Diao X, Chen Y, Wang W, Ding H. Predicting cervical lymph node metastasis in patients with papillary thyroid cancer (PTC) - Why contrast-enhanced ultrasound (CEUS) was performed before thyroidectomy. Clin Hemorheol Microcirc. 2019;72:61-73.

25. Xu SY, Yao JJ, Zhou W, Chen L, Zhan WW. Clinical characteristics and ultrasonographic features for predicting central lymph node metastasis in clinically node-negative papillary thyroid carcinoma without capsule invasion. Head \& Neck. 2019;41:3984-91.

26. Tong Y, Sun P, Yong J, Zhang H, Huang Y, Guo Y, et al. Radiogenomic Analysis of Papillary Thyroid Carcinoma for Prediction of Cervical Lymph Node Metastasis: A Preliminary Study. Front Oncol. $2021 ; 11: 682998$. 
27. Xie T, Wang X, Li M, Tong T, Yu X, Zhou Z. Pancreatic ductal adenocarcinoma: a radiomics nomogram outperforms clinical model and TNM staging for survival estimation after curative resection. Eur Radiol. 2020;30:2513-24.

28. Wu S, Zheng J, Li Y, Wu Z, Shi S, Huang M, et al. Development and Validation of an MRI-Based Radiomics Signature for the Preoperative Prediction of Lymph Node Metastasis in Bladder Cancer. EBioMedicine. 2018;34:76-84.

29. Tang P, Ren C, Shen L, Zhou Z. Development and Validation of a Diagnostic Nomogram for the Preoperative Differentiation Between Follicular Thyroid Carcinoma and Follicular Thyroid Adenomas. J Comput Assist Tomogr. 2021;45:128-34.

30. Chen B, Zhong L, Dong D, Zheng J, Fang M, Yu C, et al. Computed Tomography Radiomic Nomogram for Preoperative Prediction of Extrathyroidal Extension in Papillary Thyroid Carcinoma. Front Oncol. 2019;9:829.

31. Kwon MR, Shin JH, Park H, Cho H, Hahn SY, Park KW. Radiomics Study of Thyroid Ultrasound for Predicting BRAF Mutation in Papillary Thyroid Carcinoma: Preliminary Results. AJNR Am J Neuroradiol. 2020;41:700-5.

32. Park VY, Han K, Lee E, Kim EK, Moon HJ, Yoon JH, et al. Association Between Radiomics Signature and Disease-Free Survival in Conventional Papillary Thyroid Carcinoma. Sci Rep. 2019;9:4501.

33. Lu W, Zhong L, Dong D, Fang M, Dai Q, Leng S, et al. Radiomic analysis for preoperative prediction of cervical lymph node metastasis in patients with papillary thyroid carcinoma. Eur J Radiol. 2019;118:231-8.

\section{Figures}

\section{Figure 1}

Performance of the different models in predicting the central LNM in PTC patients. (a) ROC curves of USreported central LN status, radiomics signature, and radiomics nomogram for predicting central compartment LNM in the training cohort; (b) in the internal validation cohort; and (c-d) in two external validation cohorts. ROC, receiver operation characteristic; US, ultrasound; LN, lymph node; LNM, lymph node metastasis.

\section{Figure 2}

Performance of the different models in predicting the lateral LNM in PTC patients. (a) ROC curves of USreported lateral $L N$ status, radiomics signature, and radiomics nomogram for predicting lateral compartment LNM in the training cohort; (b) in the internal validation cohort; and (c-d) in two external 
validation cohorts. ROC, receiver operation characteristic; US, ultrasound; LN, lymph node; LNM, lymph node metastasis.

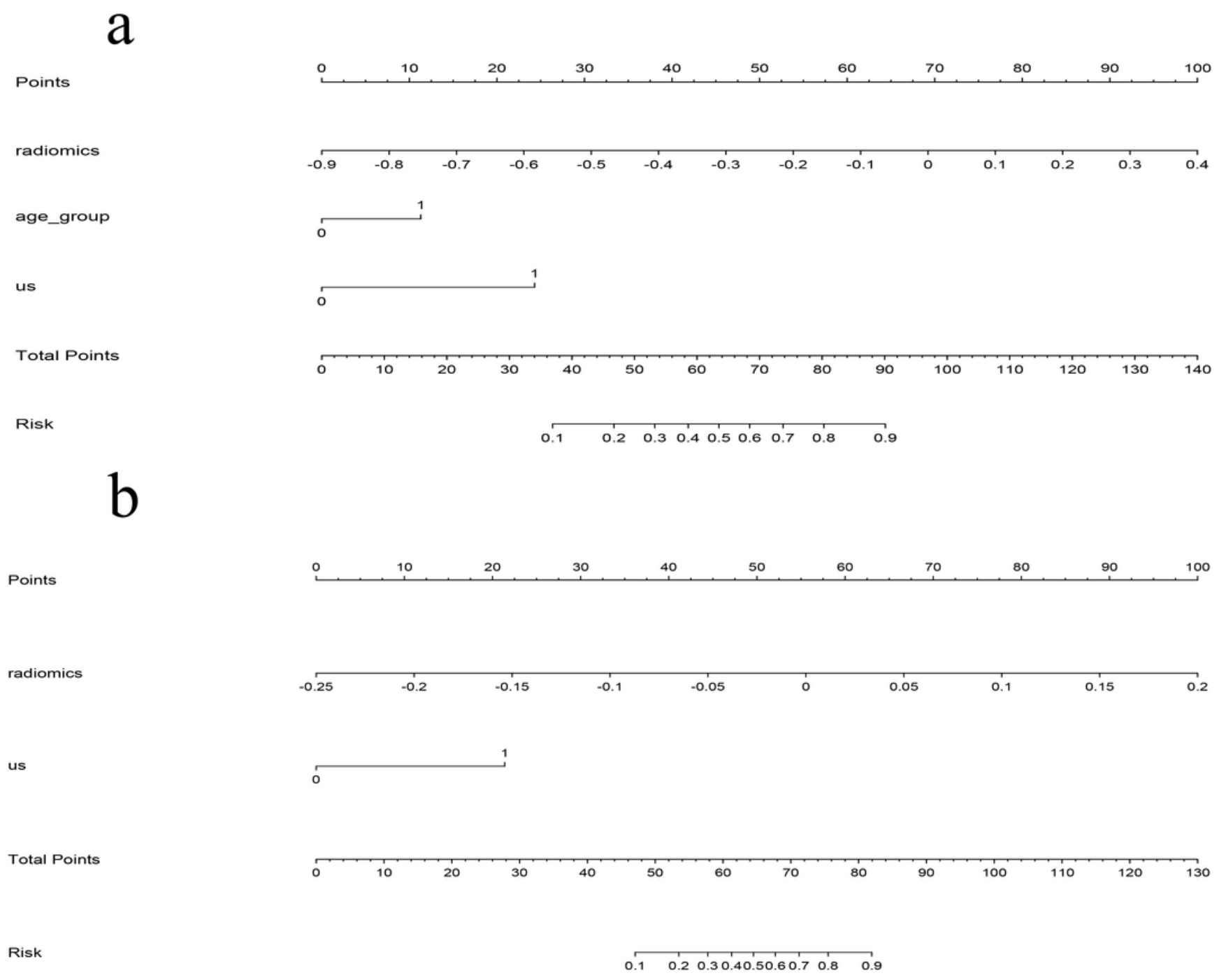

Figure 3

US-based radiomics nomograms used for prediction of (a) central compartment and (b) lateral compartment LNM in patients with PTC. US, ultrasound; LNM, lymph node metastasis; PTC, papillary thyroid carcinoma.

\section{Figure 4}

Calibration curve evaluate the radiomics nomogram used for prediction of central LNM (a) in the training cohort ( $p=0.182)$, (b) internal cohort $(0.427)$, and $(c-d)$ in two external cohorts $(p=0.561, p=0.894)$. The Hosmer-Lemeshow tests yield nonsignificant statistics. LNM, lymph node metastasis. 


\section{Figure 5}

Calibration curve evaluate the radiomics nomogram used for prediction of lateral LNM (a) in the training cohort ( $p=0.722),(b)$ internal cohort $(p=0.326)$, and $(c-d)$ in two external cohorts $(p=0.637, p=0.589)$. The Hosmer-Lemeshow tests yield nonsignificant statistics. LNM, lymph node metastasis.
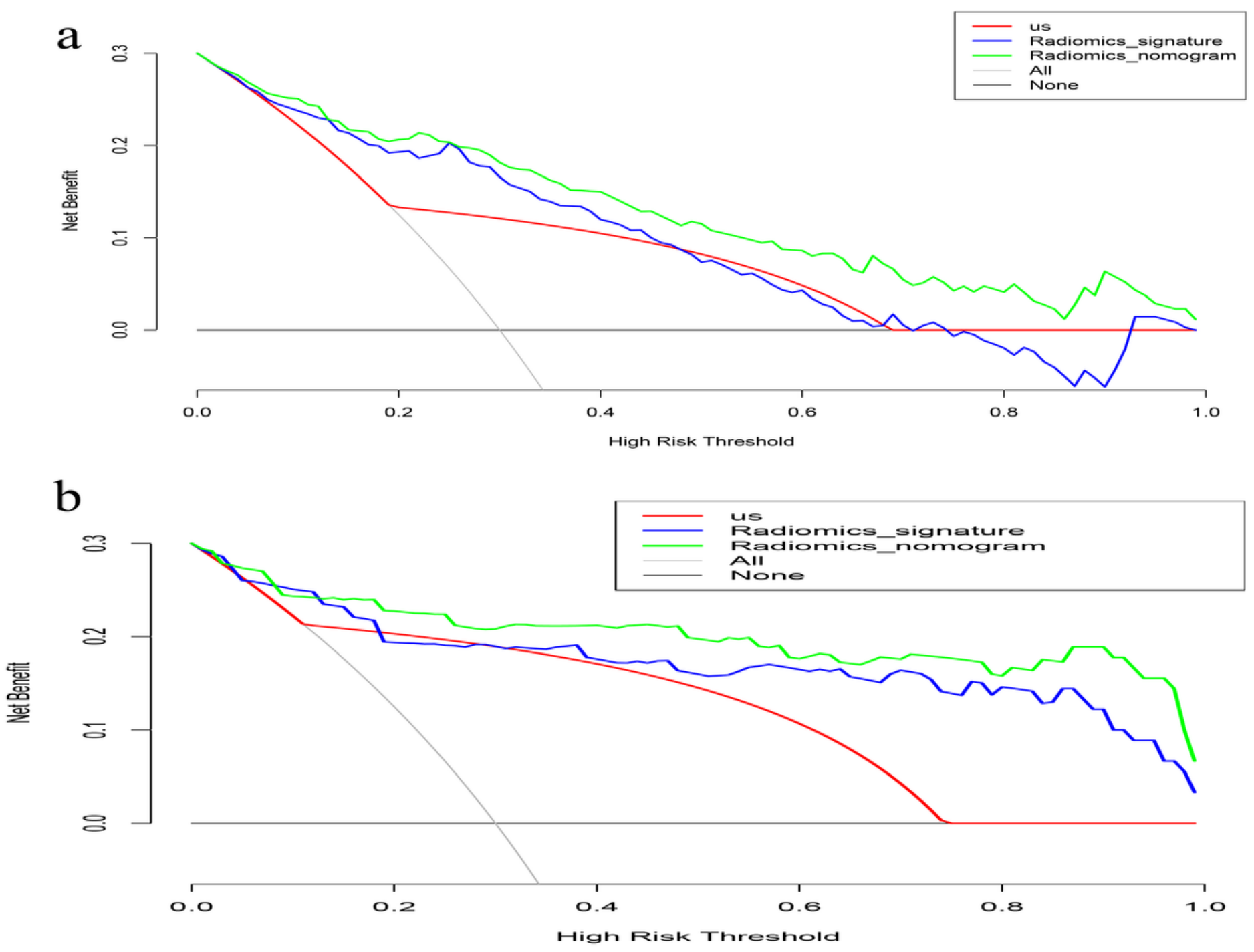

\section{Figure 6}

Decision curve analysis for each model in predicting (a) central compartment and (b) lateral compartment LNM in PTC patients. The y-axis represents the net benefit, which was calculated by summing the benefits (true positives) and subtracting the harms (false positives), and weighing the relative harm of false-positive and false-negative results. According to threshold probability obtained, the radiomics nomograms have the greatest net benefit compared with other models or simple strategies such as all-treat and non-treat scheme do. LNM, lymph node metastasis; PTC, papillary thyroid carcinoma. 